Pacific Journal of Mathematics

SUBGROuPS OF FIXED NNE 


\title{
ON SUBGROUPS OF FIXED INDEX
}

\author{
George K. White
}

If $k \in \mathscr{K}$, where $\mathscr{K}$ is a subgroup of a group $\mathscr{f}$, then closure implies $k^{2}, k^{3}, \cdots, \in \mathscr{K}$. Nonempty subsets $S \subset \mathscr{S}$ with the inverse property $s^{m} \in S$ implies $s, s^{2}, \cdots, s^{m} \in S(m=$ $1,2, \cdots$ ) will be called stellar sets. Let $p^{\alpha}$ be a fixed prime power. If a stellar set $S$ of an abelian group $\mathscr{S}$ intersects every subgroup $\mathscr{C}$ of index $p^{\alpha}$ in $\mathscr{S}$, and $0 \notin S$, then the cardinal $|S|$ of $S$ is bounded below by $p^{\alpha}$ (Theorem 3), when $\mathscr{S}$ satisfies a mild condition.

Hence for instance a subset $S$ of euclidean $n$-space $E_{n}$ intersecting all sublattices of determinant $p^{\alpha}$ of the fundamental lattice will have at least $p^{\alpha}$ elements, and more if no element is divisible by $p^{\alpha}$.

Henceforth $\mathscr{S}$ will always be an additive abelian group, so a stellar set will be one with

$$
\begin{gathered}
\varnothing \neq S \subset \mathscr{S} \\
m g \in S \Rightarrow g, 2 g, \cdots, m g \in S(g \in \mathscr{S}, m=1,2, \cdots) .
\end{gathered}
$$

Examples of stellar sets are $\mathscr{S}$ itself, and its periodic part [5, p. 137]; and a star set [7] is a symmetric stellar set. There are stellar sets of one element $s$, i.e., those $s$ for which $s=m g(m=1,2, \cdots)$ implies $m=1$. Now let $p$ be a fixed prime, and suppose $S$ intersects every subgroup $\mathscr{K}$ of $\mathscr{S}$ of index $p$. Suppose also

$$
0 \notin S
$$

(if $0 \in S$ the intersection property is redundant). Then we can say the following (in this paper we denote $|A|=$ cardinal of $A, m A=$ $\{m a ; a \in A\}$, for any set $A$ and integer $m)$ :

Theorem 1. Let $p$ be a fixed prime, $\mathscr{S}$ an abelian group, and $S$ a stellar set with $0 \notin S$ which intersects all subgroups $\mathscr{K}^{-}$of index $\mathscr{S}: \mathscr{K}=p$. Then

$$
|S| \geqq p \text {. }
$$

When $S \cap p \mathscr{S}=\varnothing$ we have $|S|>p$.

A similar result holds for ordinary sets $T$ :

THEOREM 2. Suppose $p$ is a fixed prime, $\mathscr{S}$ is an abelian group with more than one subgroup of index $p$, and $T$ is any subset of $\mathscr{S}$ with 


$$
T \cap p \mathscr{S}=\varnothing
$$

which intersects all subgroups $\mathscr{K}$ of index $\mathscr{S}: \mathscr{K}=p$. Then

$$
|T| \geqq p+1 \text {. }
$$

When $\mathscr{S}$ is the fundamental lattice $\Lambda_{0}[2,4]$ in $r$-space $E_{r}$ of all points with integral coordinates, Theorems 1 and 2 are immediate using Rogers' proof of his Theorem 1 [7] on starsets, the small adjustment needed being clear. He states his theorem with a slightly stronger hypothesis equivalent to " $S$ intersects all subgroups of index $\leqq p$ ", and for this more stringent requirement Cassels [3], replacing $p$ by $n$, has made elegant use of a generalization of Bertrand's postulate due to Sylvester [9] and Schur [8] to show $|S| \geqq n$ for $n=1,2, \cdots$ and any stellar set $S$ of an abelian $\mathscr{S}$ with no periodic part. For $n=p^{\alpha}$ a prime power we shall extend this as follows:

THEOREM 3. Suppose that $n=p^{\alpha}$ is fixed, $\mathscr{S}$ is an abelian group containing no element of order $p^{\beta}$ when $1<p^{\beta}<p^{\alpha}$, and that $S$ is a stellar set with $0 \notin S$ which intersects all subgroups $\mathscr{K}$ of index $\mathscr{S}: \mathscr{K}=p^{\alpha}$. Then

$$
|S| \geqq p^{\alpha}
$$

When $S \cap p^{\alpha} \mathscr{S}=\varnothing$, we have

$$
|S| \geqq p^{\alpha}+ \begin{cases}p & \text { if } \alpha>1 \\ 1 & \text { if } \alpha=1\end{cases}
$$

Note the requirement "contains at least one subgroup of index $p^{\alpha \text { " }}$ is a natural one, but it is an unneeded restriction on $S$. Note also that Theorem 1 is an immediate consequence of Theorem 3.

2. A lemma. We find it useful, for Rogers' case $\mathscr{S}=\Lambda_{0} \subset E^{r}$, to restate Theorem 3 in altered form. We denote $\bar{x}=\left(x_{1}, \cdots, x_{r}\right)$ so that

$$
\Lambda_{0}=\left\{\bar{x}: \text { all the } x_{i} \text { are integers, } i=1, \cdots, r\right\},
$$

and $\mathscr{S}=\Lambda_{0}$ is isomorphic to a direct sum of $r$ infinite cyclic groups. When $\bar{x} \in \Lambda_{0}$ we define $p \mid \bar{x}$ to mean $p\left|x_{1}, \cdots, p\right| x_{r}$, and

$$
\|x\|_{p}=\max \left\{\alpha: p^{\alpha} \mid \bar{x}\right\} \text {. }
$$

Let $T$ be any subset of $\Lambda_{0}$ satisfying

$$
p^{\alpha} \Lambda_{0} \cap T=\varnothing \quad\left(T \subset \Lambda_{0}\right),
$$

and a modified stellar condition 


$$
\left\{\begin{array}{l}
p^{\beta} \bar{x} \in T \text { implies } \bar{x}, 2 \bar{x}, \cdots, p^{\beta} \bar{x} \in T \\
\left(1 \leqq \beta \leqq \alpha, p^{\alpha} \text { fixed }\right)
\end{array}\right.
$$

and consider congruences

$$
\bar{l} \cdot \bar{x}=l_{1} x_{1}+\cdots+l_{r} x_{r} \equiv 0\left(p^{\alpha}\right)\left(\bar{l} \in \Lambda_{0}, p \nmid \bar{l}\right) .
$$

LEMma. If $T \subset \Lambda_{0}$ satisfies (7) and (8), $r \geqq 2$ and the congruence (9) has for each $\bar{l}$ a solution $\bar{x} \in T$, then $T$ contains at least $p^{\alpha}+p^{\min (\alpha, 2)-1}$ distinct elements $\bmod p^{\alpha}$,

$$
\left|T \bmod p^{\alpha}\right| \geqq p^{\alpha}+ \begin{cases}p & \text { if } \alpha>1 \\ 1 & \text { if } \alpha=1 .\end{cases}
$$

Proof. We consider two cases, (i) $\alpha=1$ or $r \leqq \alpha$, and (ii) $r>\alpha \geqq 2$. For the first case, a simple counting argument will suffice. Define

$$
\theta(i, \alpha)=\frac{p^{(i-1)(\alpha-1)}\left(p^{i}-1\right)}{p-1} .
$$

Then there are exactly

$$
\sum_{k=1}^{k=r} p^{(\alpha-1)(k-1)+\alpha(r-k)}=\theta(r, \alpha)
$$

distinct congruences (9), representable by

$$
\bar{l}=\left(p m_{1}, \cdots, p m_{k-1}, 1, l_{k+1}, \cdots, l_{r}\right) .
$$

If $\bar{y} \equiv b \bar{x} \bmod p^{\alpha}$ then clearly $\bar{y}$ satisfies every congruence $\bar{x}$ does, and hence we may construct a subset $V$ of $T$ which likewise satisfies every congruence (9), and also

$$
\left\{\begin{array}{l}
\bar{x} \in V, \bar{y} \in V, \bar{y} \equiv b \bar{x} \bmod p^{\alpha} \Rightarrow \bar{y}=\bar{x}, \\
\bar{x} \in V \Rightarrow \bar{x} \text { satisfies some congruence (9). }
\end{array}\right.
$$

Any $\bar{x} \in V$ may be expressed as

$$
\bar{x}=\bar{x}^{\prime} p^{\xi}\left(p \nmid \bar{x}^{\prime} ; 0 \leqq \xi=\|\bar{x}\|_{p}<\alpha\right)
$$

by (7), since $V \subset T$. A fixed $\bar{x} \in V$ obeys (9) for at least one $\bar{l}$ and in fact for precisely those $\bar{l}$ satisfying $\bar{l} \cdot \bar{x}^{\prime} \equiv 0\left(p^{\alpha-\xi}\right)$; these correspond to exactly $p^{\xi} \theta(r-1, \alpha)$ congruences (consider, e.g., $\bar{x}^{\prime}=(1$, $0, \cdots, 0)$ ). Hence, counting over the $\theta(r, \alpha)$ congruences (9), we get

$$
\theta(r, \alpha) \leqq \sum_{\bar{x} \in V} p^{\|\bar{x}\|} p \theta(r-1, \alpha) .
$$

Now $\bar{x} \in V$ obeys (8), since $V \subset T$. Hence to each $\bar{x}=\bar{x}^{\prime} p^{\xi}$ in $V$ there correspond $p^{\xi}$ elements 


$$
\Gamma(\bar{x})=\left\{\lambda \bar{x}^{\prime}: \lambda=1, \cdots, p^{\xi}\right\} \subset T \quad(\bar{x} \in V) .
$$

Moreover,

$$
\bar{x}_{1} \neq \bar{x}_{2} \text { implies } T\left(\bar{x}_{1}\right) \cap T\left(\bar{x}_{2}\right)=\varnothing \quad\left(\bar{x}_{1}, \bar{x}_{2} \in V\right),
$$

for otherwise $\lambda_{1} \bar{x}_{1}=\lambda_{2} \bar{x}_{2}, \lambda_{i}=\lambda_{i}^{\prime} p^{p i}\left(p \nmid \lambda_{i}^{\prime}\right)$, without loss of generality $\theta=\theta_{1}-\xi_{1}-\left(\theta_{2}-\xi_{2}\right) \geqq 0$, and $\lambda_{2}^{\prime} \bar{x}_{2}=\lambda_{1}^{\prime} p^{\theta} \bar{x}_{1}, \bar{x}_{2} \equiv\left(\lambda_{2}^{\prime}\right)^{-1} \lambda_{1}^{\prime} p^{\theta} \bar{x}_{1} \bmod p^{n}$, $\bar{x}_{2}=\bar{x}_{1}$ by (12). Thus by (13), (15),

$$
\begin{aligned}
& |T| \geqq \sum \sum_{\hat{x} \in V}^{p^{|x| \mid p}} \geqq \theta(r, \alpha) / \theta(r-1, \alpha) \\
& =p^{\alpha}+\frac{p^{\alpha-1}(p-1)}{p^{r-1}-1} \cdot \quad(r \geqq 2)
\end{aligned}
$$

If $\alpha=1$ we have $|T| \geqq p+(p-1)\left(p^{r-1}-1\right)^{-1}>p$, so $|T| \geqq p+1$; if $r \leqq \alpha>1$ then

$$
|T|-p^{\alpha} \geqq p^{\alpha-1}(p-1)\left(p^{\alpha-1}-1\right)^{-1}>p-1,
$$

$|T|-p^{\alpha} \geqq p$, and case (i) is verified.

For our second case $r>\alpha \geqq 2$ we employ induction on $r$. Let $r=j$, define $V \subset T$ as in case (i), and denote

$$
\bar{x}=\left(x_{1}, \cdots, x_{j-1}, x_{j}\right)=\left(\bar{x}_{0}, x_{j}\right) .
$$

There are $p^{\jmath-1}+\cdots+p+1 \geqq p^{\alpha}+p+1$ subgroups

$$
H\left(\bar{a}^{\prime}\right)=\left\{\lambda \bar{a}^{\prime} \bmod p: \lambda=1, \cdots, p \equiv 0\right\}
$$

( $\bar{a}^{\prime}$ fixed, $p \nmid \bar{a}^{\prime}$ ), any two of which intersect in a point $\bar{x}$ divisible by $p$. So if $V$ contains a primitive $(p \nmid \bar{x})$ point from each subgroup, we have $|V| \geqq p^{\alpha}+p+1$ and our result follows. Hence we may assume that $V$ does not intersect some $H\left(\bar{\alpha}^{\prime}\right)$, where without loss of generality $\bar{a}^{\prime}=(0, \cdots, 0,1)$; then $V$ contains no point of type $\bar{x}=$ $\lambda\left(p \bar{y}_{0}, 1\right) \bmod p$ when $p \nmid \lambda$, and hence by (8) no such point for any $\lambda=1,2, \cdots$,

$$
\bar{x} \in V \Rightarrow \bar{x}=p^{\beta}\left(\bar{y}_{0}^{\prime}, y_{j}\right) . \quad\left(p \nmid \bar{y}_{0}^{\prime}, 0 \leqq \beta<\alpha\right) .
$$

Now define sets $T(\bar{x})$ as in (14) and denote their union by $W$,

$$
W=\cup\{T(\bar{x}): \bar{x} \in V\},
$$

so that $V \subset W \subset S$, and $W$ is the (smallest) set generated by $V$ which satisfies the modified stellar condition (8). Denote

$$
W_{0}=\left\{\bar{x}_{0}:\left(\bar{x}_{0}, x_{j}\right) \in W \text { for some } x_{j}\right\} .
$$

Then by (17), (18), points $\bar{x}_{0}^{\prime} p^{\xi}\left(p \nmid \bar{x}_{0}^{\prime}\right)$ of $W_{0}$ correspond to points $p^{\xi}\left(\bar{x}_{0}^{\prime}, x_{j}\right)$ of $W$ and so clearly $W_{0}$ satisfies (7) and (8). But $V$ and 
hence $W$ satisfies every congruence $\bar{l}$ in (9); thus $W$ and hence $W_{0}$ satisfies every $\bar{l}$ with $l_{j}=0$ for some $\bar{x}_{0}=\left(x_{1}, \cdots, x_{j-1}\right) \in W_{0}$ such that

$$
l_{1} x_{1}+\cdots+l_{j-1} x_{j-1} \equiv 0\left(p^{\alpha}\right) \quad\left(l_{1}, \cdots, l_{j-1}, p\right)=1 .
$$

Thus by our induction hypothesis $(r=j-1, \alpha \geqq 2)$ there are at least $p^{\alpha}+p$ such $\bar{x}_{0} \in W_{0}$, and

$$
|S| \geqq|W| \geqq\left|W_{0}\right| \geqq p^{\alpha}+p .
$$

As our result is already established for $r=\alpha$ (case (i)), this completes the proof of the lemma.

3. Proof of Theorems 2 and 3. Consider the homomorphism $\eta$ :

$$
\mathscr{S} \stackrel{\eta}{\longrightarrow} \mathscr{\mathscr { S }} \cong \mathscr{S} \mid p^{\alpha} \mathscr{S}
$$

(cf. Cassels [3] for his case $s=1$ ); for Theorem 2 we take $\alpha=1$.

We see easily that if $\mathscr{S}: \mathscr{K}=p^{\alpha}$ then $p^{\alpha} \mathscr{S} \subset \mathscr{K}$ and so there is a one-to-one correspondence between all $\mathscr{K}, \mathscr{K}^{-}$of index $p^{\alpha}$ in $\mathscr{S}, \overline{\mathscr{S}}$ respectively; and any subset $V$ of $\mathscr{S}$ intersects all such $\mathscr{K}$ if and only if $\bar{V}$ intersects all such $\overline{\mathscr{K}}$ (index $p^{\alpha}$ ). If $V$ has the stellar set property this may, however, be lost under $\eta$. Since $p^{\alpha} \overline{\mathscr{S}}=0$ we have by a result of Prüfer [1] that $\overline{\mathscr{S}}$ is a direct sum of cyclic groups $C_{i}$ of orders $p^{\beta_{i}} \leqq p^{\alpha}$; in fact, $\beta_{i}=\alpha$ since in all our 3 theorems $\mathscr{P}$ has no element of order $p^{3}(0<\beta<\alpha)$ and hence $p^{\beta_{i}} c_{i}=0$ implies $\beta_{i} \geqq \alpha$. Thus

$$
\overline{\mathscr{S}}=\sum_{i \in I}^{\oplus} C_{i}\left(C_{i} \cong\left\langle e: p^{\alpha} e=0\right\rangle\right) .
$$

Note that all $s \in S$ have infinite period,

$$
m s \neq 0 \quad(s \in S, m= \pm 1, \pm 2, \cdots)
$$

since otherwise $|m| s=0, s=(|m|+1) s \in S$ so $0=|m| s \in S$ contrary to (2). Now suppose $\overline{0} \in \bar{S}$. Then $p^{\alpha} g \in S$ so $g, 2 g, \cdots, p^{\alpha} g \in S,|S| \geqq p^{\alpha}$ since otherwise $i g=j g(i<j)$ and $g \in S$ has finite period. It remains therefore to settle the matter when

$$
\left.\overline{0} \notin \bar{S} \quad \text { (i.e., } S \cap p^{\alpha} \mathscr{S}=\varnothing\right) \text {. }
$$

The cases $|I|=0,1$ in (20) correspond to groups $\mathscr{P}$ with no, exactly one subgroup of index $p^{\alpha}$. In the latter event we have $\overline{0} \in \bar{S}$, a case already settled. If $|I|=0$ in Theorem 3 then $\mathscr{S}=p^{x} . \mathscr{S}$ and all stellar sets $S$ vacuously satisfy the intersection condition. No stellar set is empty, so we have $s \in S, s=p^{\alpha} s_{1}, s_{1}=p^{\alpha} s_{2}, \cdots$, and $|S|=\infty$ since otherwise $s_{i}=s_{j}(i<j)$ and $s_{j} \in S$ has finite period, contrary to (21). 
The case $|I| \leqq 1$ does not occur for Theorem 2 , since here $\mathscr{S}$ has $\geqq 2$ subgroups of index $p^{\alpha}$. Hence we may assume

$$
|I| \geqq 2 \text {. }
$$

From (23) it is immediate that $\mathscr{S}$ contains more than one subgroup of index $p^{\alpha}$. We consider only Theorem 3 from now on; Theorem 2 will follow by the same reasoning $(\alpha=1)$.

It remains, then, to verify Theorem 3 when (22), (23) hold. Assume now then

$$
|S|<\infty,
$$

since if $|S|=\infty$ we have nothing to prove. Then if we decompose $\bar{s}=\sum_{s_{i}}$ in (20) we have $s_{i} \neq 0$ for some $\bar{s} \in \bar{S}$ for only a finite number of $i \in I$, which we may include in a finite set $i=1, \cdots, j(2 \leqq j \leqq|I|)$. Then

$$
\begin{gathered}
\bar{S} \subset \mathscr{S}^{(0)} \cong \Lambda_{0} \bmod p^{\alpha} \quad\left(\text { in } j \text {-space } E^{j}\right), \quad(2 \leqq j), \\
\overline{\mathscr{S}}=\mathscr{S}^{(0)} \oplus \mathscr{S}^{*},
\end{gathered}
$$

and we may represent any $\bar{x} \in \overline{\mathscr{S}}$ uniquely by

$$
\bar{x}=x^{(0)}+x^{*}=\left(x_{1}, \cdots, x_{j} ; x^{*}\right) \bmod p^{\alpha} .
$$

The following subgroups $\overline{\mathscr{K}}$ have index $p^{\alpha}$ in $\overline{\mathscr{S}}$ and hence are intersected by $\bar{S}$ :

$$
\overline{\mathscr{K}}=\left\{\bar{x}: l_{1} x_{1}+\cdots+l_{j} x_{j} \equiv 0\left(p^{\alpha}\right)\right\} \quad\left(l_{1}, \cdots, l_{j}, p\right)=1,
$$

where $\left(l_{i}, p\right)=1$ for some $i$ and $l_{1}, \cdots, l_{j}$ are fixed for each $\overline{\mathscr{K}}$ (cf. [3, preceding (10)]); we have $p \nmid l_{i}$ for at least one $i$ and so for each $\bar{x} \in \overline{\mathscr{K}}, x_{i}=-\sum_{j \neq i} l_{i}^{-1} l_{j} x_{j}$. Hence $\left|\mathscr{K}_{0}\right|=p^{\alpha(j-1)}$,

$$
\mathscr{S}: \mathscr{K}=\mathscr{S}_{0}: \mathscr{K}_{0}=p^{\alpha j} / p^{\alpha(j-1)}=p^{\alpha} \text {. }
$$

Elements $\bar{s}$ of $\bar{S}$ are of type $\bar{s}=\left(s_{1}, \cdots, s_{j} ; 0^{*}\right)$; since $S$ is a stellar set the modified property (8) holds for $T=\bar{S}$; also, $0=\left(0, \cdots, 0,0^{*}\right) \notin \bar{S}$ and $r=j \geqq 2$ by (22), (23). So we may apply the lemma to find there are at least $p^{\alpha}+p^{\min (\alpha, 2)-1}$ distinct points $\left(s_{1}, \cdots, s_{j}, 0^{*}\right)$ in $\bar{S}$; hence

$$
|S| \geqq|\bar{S}| \geqq p^{\alpha}+p^{\min (\alpha, 2)-1},
$$

and our proof of Theorems 2,3 is complete.

4. Remarks. 1. In our proof of Theorem 3 we utilize the stellar property of $S$ only through its consequence in $\bar{S}$, a condition of type (8) with $T=\bar{S}$ which would clearly follow from imposing 
condition (8) on $S$, along with $S \neq \varnothing$. Hence we may make the following extension:

THEOREM 4. Theorem 3 holds for $S$ not a stellar set, if $S$ satisfies (8) $(T=S \subset \mathscr{S}, \bar{x} \in \mathscr{S})$, and $S \neq \varnothing$.

2. When $\mathscr{S}$ is not abelian, Theorems 1-4 need not hold; e.g., the direct sum $\mathscr{S}=C^{\infty} \oplus A_{5}$ of the infinite cyclic group and alternating group of 60 elements has only one subgroup of index $3, \mathscr{K}^{-}=$ $3 C^{\infty} \oplus A_{5}$, and $\mathscr{K}$ is intersected by the stellar set of one element,

$$
S=\{3+\text { cycle }(123)\} \neq 3 g \text {. }
$$

3. In the excluded case $0 \in S$ the least stellar set containing 0 is the periodic part of $\mathscr{S}$, and $|S| \geqq p$ need not follow.

4. When $\mathscr{S}=\Lambda_{0}(r \geqq 2)$, the set of all $\left(1, x_{2}, 0, \cdots, 0\right),\left(p x_{1}, 1,0\right.$, $\cdots, 0) \bmod p^{\alpha}$ is a stellar set of $p^{\alpha}+p^{\alpha-1}$ elements intersecting all congruences (9) $\bmod p^{\alpha}$. So our bounds are best possible, for the lemma, when $\alpha=1,2$. $(r \geqq 2)$.

5. In Theorem 3 we must exclude elements of order $p^{\beta}(\beta<\alpha)$. For consider ,e.g., $\mathscr{S}=C^{\infty} \oplus C^{(p)}$ (any $\alpha$ ). Here the bound is $p^{\alpha}+1$.

6. Let $\alpha \geqq 2, S$ be a stellar set in Euclidean $n$-space $\{\bar{x}=$ $\left.\left(x_{1}, \cdots, x_{n}\right)\right\}$ with fewer than $p^{\alpha}+p$ elements, and no element $p^{\alpha} \bar{x}$. Then there is a sublattice of the fundamental lattice of determinant $p^{\alpha}$ (see [2], p. 10) which is not intersected by $S$.

7. Our condition $(A)^{\prime \prime} S$ intersects all subgroups of index $n^{\prime \prime}$ is equivalent to $(B)^{\prime \prime} \ldots$ index $d: d \mid n^{\prime \prime}$ though weaker than $(C)^{\prime \prime} \ldots$ index $m: m<n^{\prime \prime}$. The latter remark follows from the example $S=$ $\{(4,1),(2,1),(2,0),(1,0)\}$ in $\mathscr{S}=C^{\infty} \oplus C^{(2)}(n=4)$. For the former prove first for $d=n / p$ and then iterate: if $\mathscr{S}: \mathscr{C}=n / p(p \mid n)$ and (A) holds then $\mathscr{C} \neq p \mathscr{K}$, there exist $\mathscr{C}$ in $\mathscr{C}$ with $\mathscr{H}: \mathscr{C l}=p$ so $\mathscr{S}: \mathscr{C l}=n, \mathscr{K} \cap S \neq \varnothing$.

8. Theorem 3 does not hold for all $n=1,2, \cdots$. Mr. George M. Bergman of Cambridge, Mass. has kindly furnished me with a set of counterexamples for $\mathscr{S}=C^{\infty} \oplus C^{\infty}$, which includes a stellar set $S$ of 76 elements that intersects every subgroup of index 77 .

9. Finally, we should like to acknowledge here some parallel though independent work of $\mathrm{Mr}$. Bergman who in unpublished cor- 
respondence proves a simpler version of Theorem 4, obtaining a slightly lower bound ( $p^{\alpha}$ rather than $\left.p^{\alpha}+p, 1\right)$. His proof is in essence similar to ours, except there is no induction step: a homomorphism $\eta$ (19) reduces the problem to Rogers' case $\mathscr{S}=\Lambda_{0}$, and a version of our lemma is proved by arguments resembling ours for $\alpha=1$ or $r \geqq \alpha$, Mr. Bergman in effect considering congruences (9) with $l_{1}=1$ to obtain his bound $p^{\alpha}$ for (10) for all $r, \alpha$, without induction. We thank Mr. Bergman for the material communicated; among other things it helped remind us to include Theorem 4. We thank him also for welcome suggestions concerning our final draft.

\section{REFERENCES}

1. J. L. Brenner, The decomposition theorem for abelian groups Bull. Amer. Math. Soc. 47 (1941), 116-117.

2. J.W.S. Cassels, An introduction to the geometry of numbers Grund d. Mat. Wiss 99 (Berlin, 1959).

3. - On the subgroups of infinite abelian groups, J. London Math. Soc. 33 (1958), 281-284.

4. G. H. Hardy and E. M. Wright, An introduction to the theory of numbers, Oxford, 1954.

5. A. C. Kurosh, The theory of groups I (Trans. K. A. Hirsch), Chelsea, New York, 1955.

6. H. Prüfer, Untersuchungen ïber die Zerlegbarkeit der abzählbaren primären Abelschen Gruppen, Math. Zeit. 17 (1923), 35-61.

7. C. A. Rogers, The number of lattice points in a star body J. London Math. Soc. 26 (1951), 307-310.

8. I. Schur, Einige Sätze ïber Primzahlen mit Anwendungen auf Irreduzibilitätsfragen I, II Sitzungsberichte der Preuss. Akad. Wiss. (1929), 125-136, 370-391.

9. J. Sylvester, On arithmetical series, Mess of Math. 21 (1892), 1-19, 87-120. (Reprinted in Collected Works IV, 687-731.)

Received October 13, 1967.

UNIVERSITY OF BRITISH COLUMBIA AND

Dalhousie UNIVERSity 


\section{PACIFIC JOURNAL OF MATHEMATICS}

\section{EDITORS}

H. ROYDEN

Stanford University

Stanford, California

R. R Phelps

University of Washington

Seattle, Washington 98105
J. DUGUNDJI

Department of Mathematics

University of Southern California

Los Angeles, California 90007

RICHARD ARENS

University of California

Los Angeles, California 90024

\section{ASSOCIATE EDITORS}
E. F. BECKENBACH
B. H. NeumanN
F. WOLF
K. YosidA

\section{SUPPORTING INSTITUTIONS}

UNIVERSITY OF BRITISH COLUMBIA

CALIFORNIA INSTITUTE OF TECHNOLOGY

UNIVERSITY OF CALIFORNIA

MONTANA STATE UNIVERSITY

UNIVERSITY OF NEVADA

NEW MEXICO STATE UNIVERSITY

OREGON STATE UNIVERSITY

UNIVERSITY OF OREGON

OSAKA UNIVERSITY

UNIVERSITY OF SOUTHERN CALIFORNIA
STANFORD UNIVERSITY

UNIVERSITY OF TOKYO

UNIVERSITY OF UTAH

WASHINGTON STATE UNIVERSITY

UNIVERSITY OF WASHINGTON

AMERICAN MATHEMATICAL SOCIETY CHEVRON RESEARCH CORPORATION TRW SYSTEMS

NAVAL WEAPONS CENTER 


\section{Pacific Journal of Mathematics

Vol. 28, No. $1 \quad$ March, 1969

Patrick Robert Ahern, On the geometry of the unit ball in the space of real annihilating measures .............................. 1

Kirby Alan Baker, Equational classes of modular lattices ............. 9

E. F. Beckenbach and Gerald Andrew Hutchison, Meromorphic minimal surfaces ......................................... 17

Tae Ho Choe, Intrinsic topologies in a topological lattice ..............

John Bligh Conway, A theorem on sequential convergence of measures and

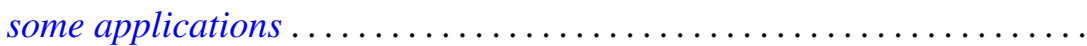

Roger Cuppens, On the decomposition of infinitely divisible probability laws without normal factor.

Lynn Harry Erbe, Nonoscillatory solutions of second order nonlinear

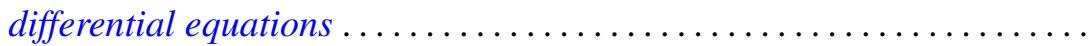

Burton I. Fein, The Schur index for projective representations of finite

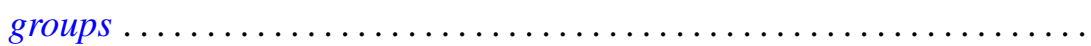

Stanley P. Gudder, A note on proposition observables............... 101

Kenneth Kapp, On Croisot's theory of decompositions ............... 105

Robert P. Kaufman, Gap series and an example to Malliavin's theorem . . . 117

E. J. McShane, Robert Breckenridge Warfield, Jr. and V. M. Warfield,

Invariant extensions of linear functionals, with applications to measures and stochastic processes ................

Marvin Victor Mielke, Rearrangement of spherical modifications ...

Akio Osada, On unicity of capacity functions ..............

Donald Steven Passman, Some 5/2 transitive permutation groups ...

Harold L. Peterson, Jr., Regular and irregular measures on groups and dyadic spaces...

Habib Salehi, On interpolation of $q$-variate stationary stochastic processes...

Michael Samuel Skaff, Vector valued Orlicz spaces generalized

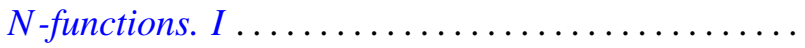

A. J. Ward, On $H$-equivalence of uniformities. II...........

Thomas Paul Whaley, Algebras satisfying the descending chain condition for subalgebras...

G. K. White, On subgroups of fixed index

Martin Michael Zuckerman, A unifying condition for implications among the axioms of choice for finite sets ................. 